\title{
Spin polarized photoelectrons from atoms and molecules
}

\author{
U. Heinzmann
}

\begin{abstract}
This paper reviews the experimental activities since 1979 for the study of the spin polarization of photoelectrons emitted by atoms and molecules exposed to VUV radiation. Two series of studies have been performed: First, using unpolarized and linearly polarized radiation the photoionization of the rare gases $\mathrm{Ar}$, $\mathrm{Kr}$, and $\mathrm{Xe}$ has been studied in an angle-, energy-, and spin-resolved photoemission experiment. Second, the spin polarization of photoelectrons produced by circularly polarized VUV radiation has been measured for some atoms and molecules. This was performed for most targets by use of the synchrotron radiation in Bonn.
\end{abstract}

\section{Introduction}

One novelty in photoionization is the fact that photoelectrons ejected from unpolarized atoms can be spin polarized, if the light is circularly polarized or even unpolarized. It is necessary to know these spin polarization values to determine the matrix elements of the dipole operator as well as the difference between the phases of the continuum wave functions. Photoionization cross section, angular distribution, and spin polarization experiments complement each other in reaching a full understanding of the photoionization process.

Cherepkov ${ }^{1}$ and $\mathrm{Lee}^{2}$ have pointed out that the photoionization process arising from the outermost subshell for most atoms (alkalis and rare gases, for example) is described by five parameters, which are functions of the photon energy and the kinetic energy of the photoelectrons. These five parameters can be determined by five types of measurement.

In the first three types of experiment unpolarized radiation may be used: tion;

(1) measurement of the photoionization cross sec-

(2) determination of the asymmetry parameter $\beta$ describing the angular distribution of the differential cross section;

(3) measurement of the spin polarization of photoelectrons $\left.P(\theta)=\left(N_{\uparrow}-N_{\downarrow}\right) / N_{\uparrow}+N_{\downarrow}\right)$, where $N_{\uparrow}$ and $N_{\downarrow}$ are the numbers of electrons produced with spin up and

The author is with Physikalisches Institut der Universität Münster, 4400 Münster, Federal Republic of Germany.

Received 6 June 1980.

0003-6935/80/234087-05\$00.50/0.

(C) 1980 Optical Society of America. spin down, perpendicular to the reaction plane given by the directions of incoming photon and outgoing electron. $N_{\uparrow}-N_{\downarrow}$ is proportional to $\xi \cdot \sin \theta \cos \theta$, where $\theta$ is the angle between the two directions described above, and the parameter $\xi$ determines the size of $P$.

In the fourth and fifth experiments, where circularly polarized radiation has to be used, one observes the spin transfer from the photon to the electron:

(4) measurement of the average spin polarization (parallel or antiparallel to the photon spin) if all the photoelectrons produced are extracted by an electric field regardless of their direction of emission (Fano effect $\left.^{3}\right)$;

(5) determination of the parameter $\alpha$ describing the angular dependence of the photoelectron polarization in the direction of the photon spin transferred.

It is the purpose of this paper to give a survey of the polarization experiments of types (3) and (4) performed in the past year and to show, using the photoionization of $\mathrm{Xe}$, what can be learned from the results of these experiments.

\section{Angle-, Energy-, and Spin-Resolved Photoemission Experiments of Rare Gas Atoms Exposed to Unpolarized Radiation}

Spin polarization of photoelectrons ejected by unpolarized light from unpolarized targets was first found in lead atoms. ${ }^{4}$ But in contrast to the lead experiment where the photoelectron emission studied was only angle- and spin-resolved, in the experiments on rare gases $^{5,6}$ the analysis of the photoelectrons has also been resolved with respect to the photon energy and the kinetic energy of the photoelectrons.

The complication in such polarization measurements is that they combine the intensity problem of photoelectron spin analysis with the difficulties of photoelectron angular- and energy-distribution experiments. 


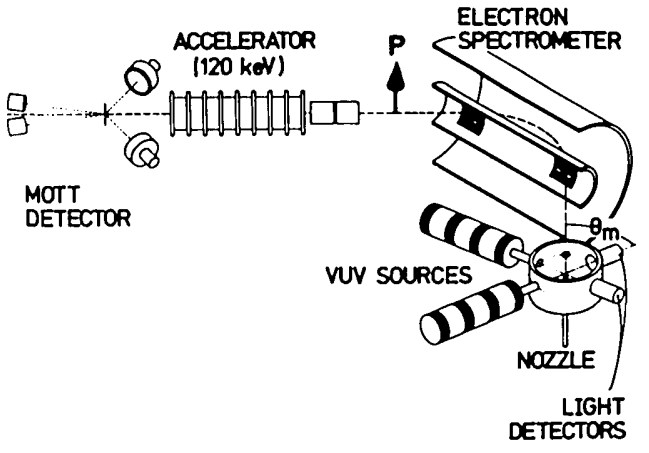

Fig. 1. Schematic diagram of the apparatus for the measurement of $\xi$.

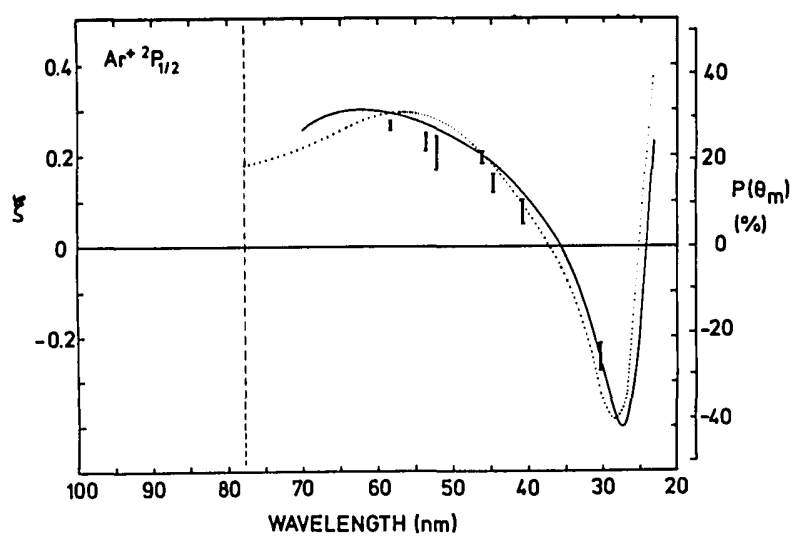

(a)

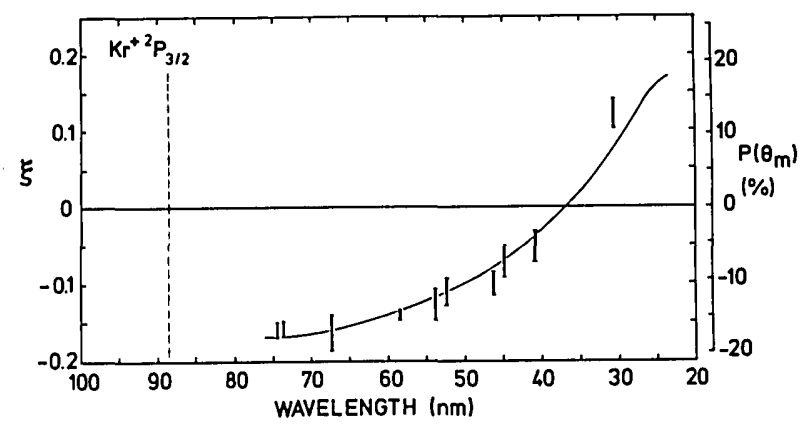

(d)

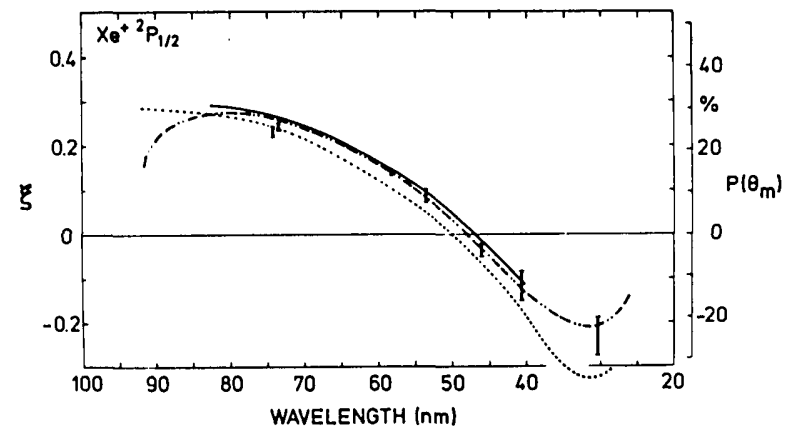

(e)
Therefore, it was necessary to build special capillary discharge tubes ${ }^{7}$ yielding very intense $\mathrm{He}, \mathrm{Ne}$, and $\mathrm{Ar}$ resonance lines (He I: $8 \times 10^{12}$ photons $\mathrm{sec}^{-1}$ ). A schematic diagram of the apparatus used is shown in Fig. 1. The VUV radiation crosses the atomic beam in a region free of electric and magnetic fields. The photoelectrons produced pass through an electron spectrometer (CMA) and are accelerated to an energy of 120 $\mathrm{keV}$ for analysis of spin polarization, determined by the left-right asymmetry of the electron intensity scattered through $120^{\circ}$ by the gold foil of the Mott detector.

In contrast to the photoionization experiment using linearly polarized radiation, ${ }^{8}$ in which the full angular dependence of the polarization $P(\theta)$ has been measured

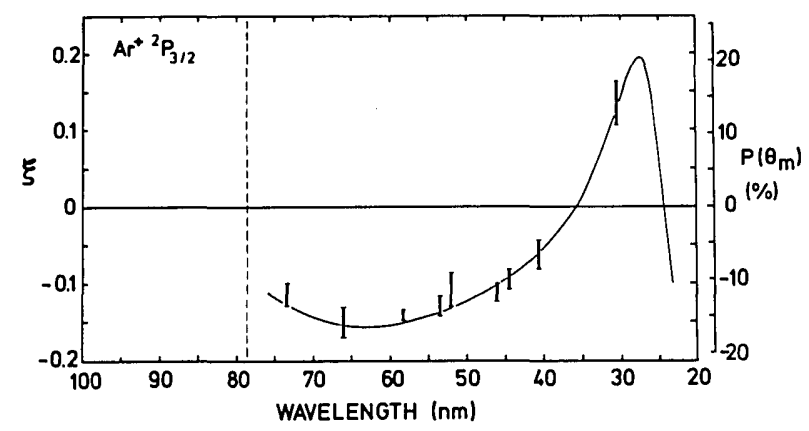

(b)

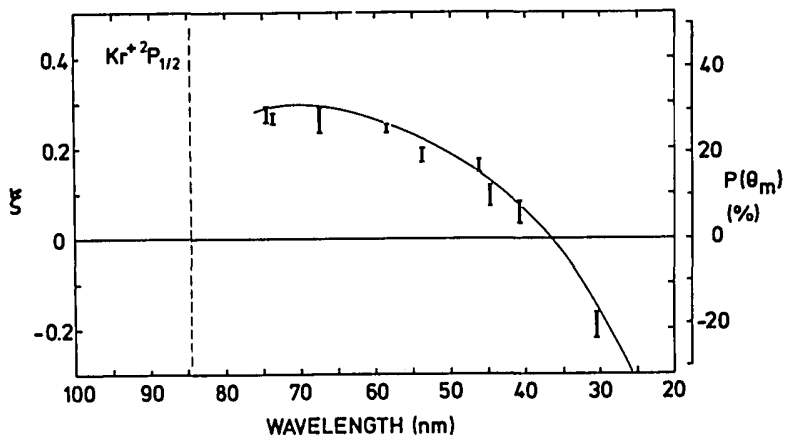

(c)

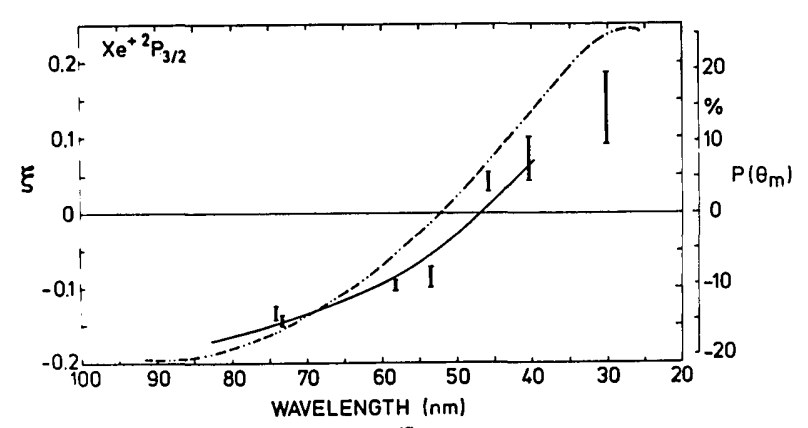

(f)

Fig. 2. Experimental results (error bars) of photoelectron polarization produced by unpolarized radiation (right-hand scale) and for parameter $\xi$ (left-hand scale). Curves follow from calculations using RRPA $(-9)$, RPAE $\left(--^{10}\right)$, and MQDT $(-\cdots-, 11,12)$ : (a) for photoelectrons corresponding to the ionic state ${ }^{2} P_{1 / 2}$ of $\mathrm{Ar}^{6}$; (b) for photoelectrons corresponding to the ionic state ${ }^{2} P_{3 / 2}$ of $\mathrm{Ar}^{6}$; (c) for photoelectrons corresponding to the ionic state ${ }^{2} P_{1 / 2}$ of $\mathrm{Kr}^{6}$; (d) for photoelectrons corresponding to the ionic state ${ }^{2} P_{3 / 2}$ of $\mathrm{Kr}^{6}$; (e) for photoelectrons corresponding to the ionic state ${ }^{2} P_{1 / 2}$ of $\mathrm{Xe}^{5,11}$; (f) for photoelectrons corresponding to the ionic state ${ }^{2} P_{3 / 2}$ of $\mathrm{Xe} .5,11$ 
(verifying the $\sin \theta \cdot \cos \theta$-proportionality mentioned above) in the experiment using unpolarized radiation (Fig. 1), the angles $\theta$ are the magic angles $\pm \theta_{m}= \pm$ $54^{\circ} 44^{\prime}$, because at these angles the determination of $\xi$ is independent of the knowledge of the asymmetry parameter $\beta\left[\xi=1.061 \cdot P\left(\theta_{m}\right)\right]$.

Figure 2 shows the parameter $\xi$ and the polarizations measured as functions of the wavelength for $\mathrm{Ar}, \mathrm{Kr}$, and Xe. The polarization of photoelectrons associated with the ${ }^{2} P_{1 / 2}$ and ${ }^{2} P_{3 / 2}$ states of the residual ions differs in sign for all atoms, which shows the necessity of resolving the fine structure by use of the electron spectrometer.

The experimental results given in Fig. 2 show, within the error limits, good agreement with the theoretical curves. In Fig. 2 full lines ${ }^{9}$ are calculated using the relativistic random phase approximation (RRPA), dotted lines ${ }^{10}$ are calculated using the random phase approximation with exchange (RPAE), and dasheddotted curves are calculated using multichannel quantum defect theory (MQDT) ${ }^{11}$ taking the experimental data from Ref. 12.

It is worth noting that the magnitude of the electron polarization does not depend on whether the spin-orbit interaction, which is responsible for the existence of the polarization, is strong or weak but only on whether the ionic fine structure splitting is resolved. While this fine structure splitting is seven times larger in Xe than in Ar, the magnitudes of the polarization measured are nearly the same for $\mathrm{Ar}, \mathrm{Kr}$, and $\mathrm{Xe}$ (see Fig. 2).

\section{Fano Effect with Circularly Polarized Synchrotron Radiation}

Experimental studies of the polarization of photoelectrons produced by circularly polarized radiation are hampered by the fact that most atoms and molecules have their ionization thresholds in the VUV, where conventional methods for producing circularly polarized radiation break down. Such experiments can, however, be performed with synchrotron radiation. The synchrotron emission is linearly polarized in the plane of the synchrotron, but above and below the plane it is largely circularly polarized.

A schematic diagram of the apparatus, ${ }^{11,13}$ built at the 2.5-GeV synchrotron in Bonn, is shown in Fig. 3. A $10-\mathrm{m}$ normal incidence monochromator with a plane holographic grating (4960 lines $\mathrm{mm}^{-1}$ ) and a concave mirror that produces a one-to-one image of the electron beam in the exit slit was built. The radiation coming from the electron beam (within an accepted horizontal angle of $22 \mathrm{mrad}$ ) is cut off in the vertical direction by an aperture, which is able to move up and down for selecting radiation of left- or right-handed circular polarization.

The radiation coming through the exit slit had a bandwidth of $0.05 \mathrm{~nm}$. After passing through the atomic beam, it was analyzed polarimetrically by successive reflections from four gold mirrors (shown in Fig. 3 ) in a rotatable analyzer, or its absolute intensity was

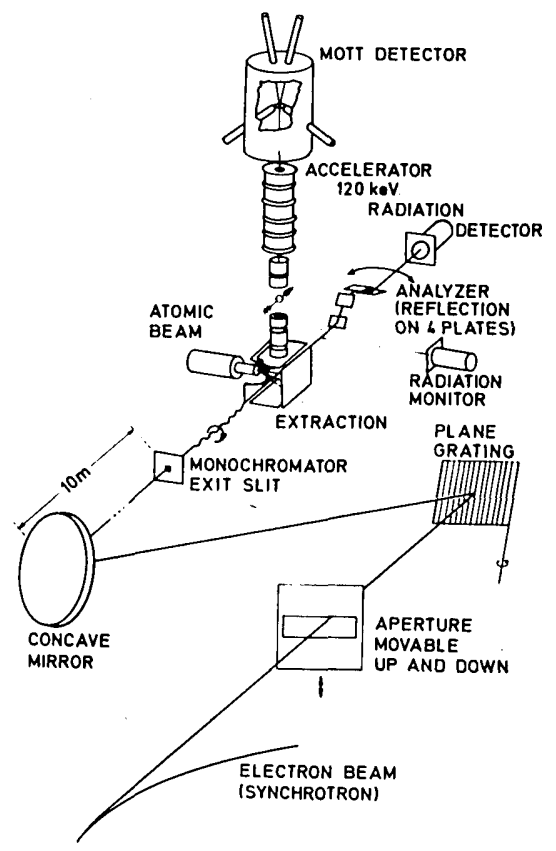

Fig. 3. Schematic diagram of the apparatus for the measurement of the Fano effect.

measured by a double ionization chamber of the Samson ${ }^{14}$ type (not shown in Fig. 3.) The circular polarization of the radiation emitted into an angular vertical range from 1 to $3.5 \mathrm{mrad}$ with respect to the synchrotron plane has been measured ${ }^{11,13}$ to be $-83 \pm 3 \%$ for the spectral range from 50 to $100 \mathrm{~nm}$. The intensity of the circularly polarized radiation was measured there ${ }^{15}$ to be between $10^{9}$ and $10^{10}$ photons $\sec ^{-1}$.

The photoelectrons produced were extracted by an electric field regardless of their directions of emission and accelerated to $120 \mathrm{keV}$ for spin polarization analysis in a Mott detector.

Using this apparatus experiments on $\mathrm{Ar}, \mathrm{Kr},{ }^{16}$ and $\mathrm{Xe}^{13}$ atoms as well as on $\mathrm{CO}_{2}$ and $\mathrm{N}_{2} \mathrm{O}$ molecules ${ }^{17}$ have been performed. Figure 4 shows the measured values of spin polarization and photoelectron intensity (error bars and points) for the autoionization range of $\mathrm{Kr}$. The good agreement of the photoelectron intensity measured compared with the experimental results of another author ${ }^{18}$ (solid line in Fig. 4) indicates that the bandwidth of the radiation used was adequate even for observations of the narrow resonances. Furthermore, this provided an additional calibration of the $10-\mathrm{m}$ monochromator used. The measured polarization (given in the lower part of Fig. 4) also shows a pronounced resonance structure due to autoionization processes.

In Fig. 5, where the results obtained in the autoionization range of $\mathrm{Xe}$ are given, a comparison between the experimental results (full curves, connecting the black rectangles of error bars) and theoretical curves calculated by means of the MQDT (dashed ${ }^{2}$ and dotted ${ }^{11,12}$ curves) shows a discrepancy between theory and experiment for the position of the resonances, although 
Fig. 4. Photoionization of krypton atoms in the autoionization range: (top) cross section (photoelectron intensity) measured; data taken from Ref. $16(\bullet)$ and Ref. 18 (-); (bottom) spin polarization of photoelectrons. ${ }^{16}$
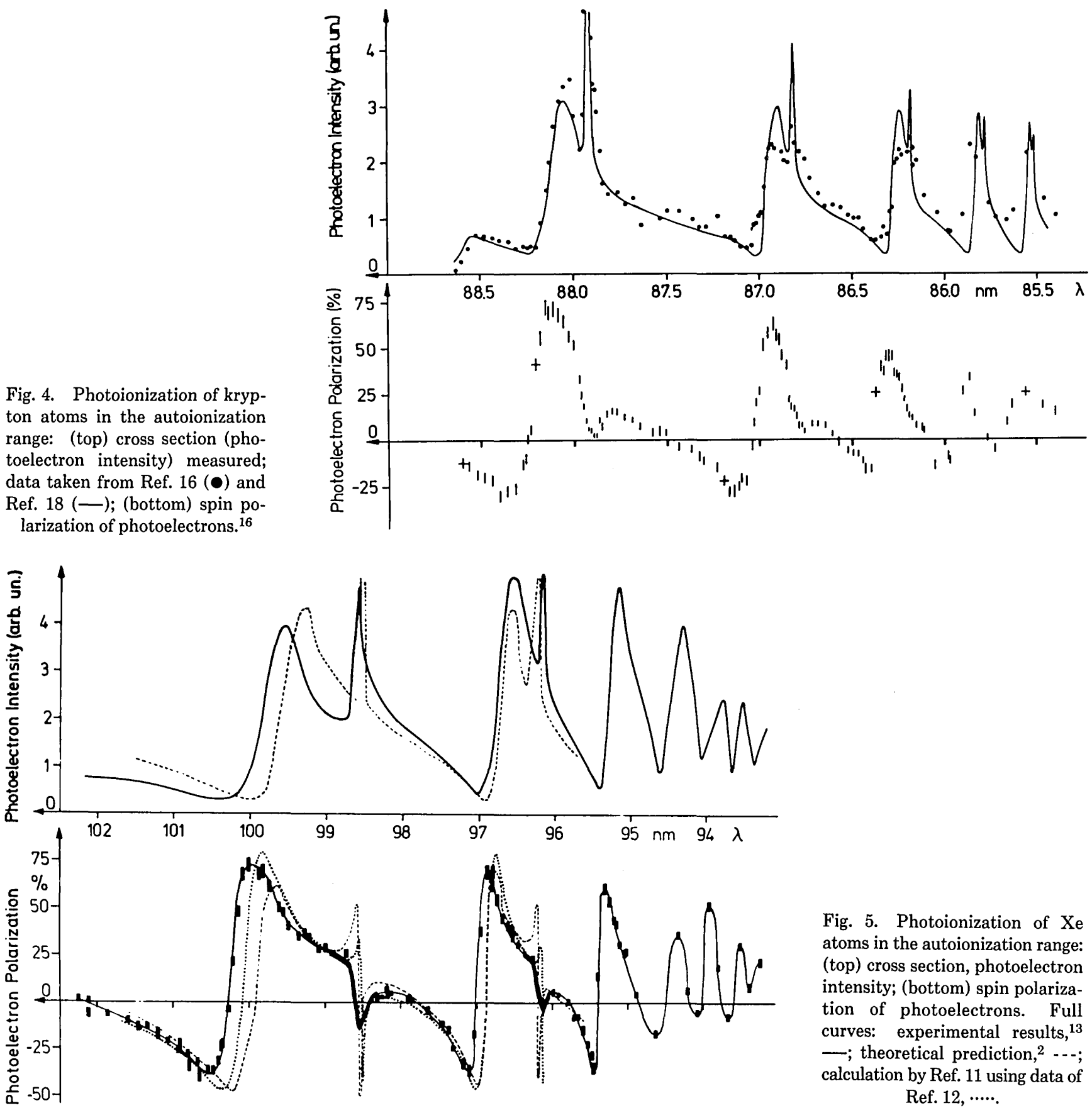

Fig. 5. Photoionization of $\mathrm{Xe}$ atoms in the autoionization range: (top) cross section, photoelectron intensity; (bottom) spin polarization of photoelectrons. Full curves: experimental results, ${ }^{13}$ -; theoretical prediction, ${ }^{2}-{ }^{-- \text {; }}$ calculation by Ref. 11 using data of Ref. $12, \cdots .$.

the shapes of the curves are very similar. This discrepancy is also seen in the comparison of the calculated photoionization cross section with all the measured value of different authors. ${ }^{13,18,19}$

Figure 6 (bottom) shows, using as an example $\mathrm{N}_{2} \mathrm{O}$, that photoelectrons emerging from molecules can also be spin polarized. ${ }^{17}$ Similar to the case of $\mathrm{CO}_{2}$ the polarization occurs at the photoionization threshold the total photoelectron intensity drops monotonically there ${ }^{20}$ [see Fig. 6 (top)], if there is an ionic fine structure splitting due to spin-orbit interaction [Fig. 6 (center) $\left.{ }^{21}\right]$.

It is worth noting that polarized electrons have also been found in the past months in $\mathrm{Ag}$ atoms ${ }^{22}$ in an ex- periment using a conventional radiation source and an $\mathrm{MgF}_{2}$ circular polarizer ${ }^{23}$ in the wavelength range between 150 and $160 \mathrm{~nm}$.

\section{Conclusion}

As pointed out in Sec. I, spin polarization results are needed in addition to the cross section values and the angular distribution to reach a complete understanding of the photoionization process. To show that it is possible in, for example, Xe to determine experimentally all matrix elements of the dipole operator as well as the phase differences of the continuum wave functions, in Fig. 7 the results for a certain bound-free transition are given: $\mathrm{A}$ bound $p_{1 / 2}$ electron is able to 


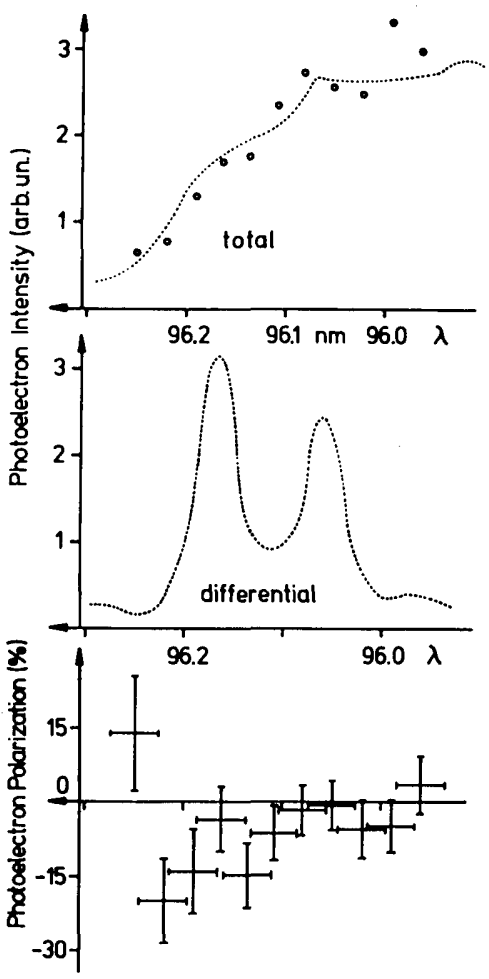

Fig. 6. Photoionization of $\mathrm{N}_{2} \mathrm{O}$ at the threshold: (top) measured photoelectron intensity $\left(0,{ }^{17} \ldots . .,{ }^{20}\right)$; (center) differential photoelectron spectrum measured by Ref. 21; (bottom) measured spin polarization of photoelectrons. ${ }^{17}$

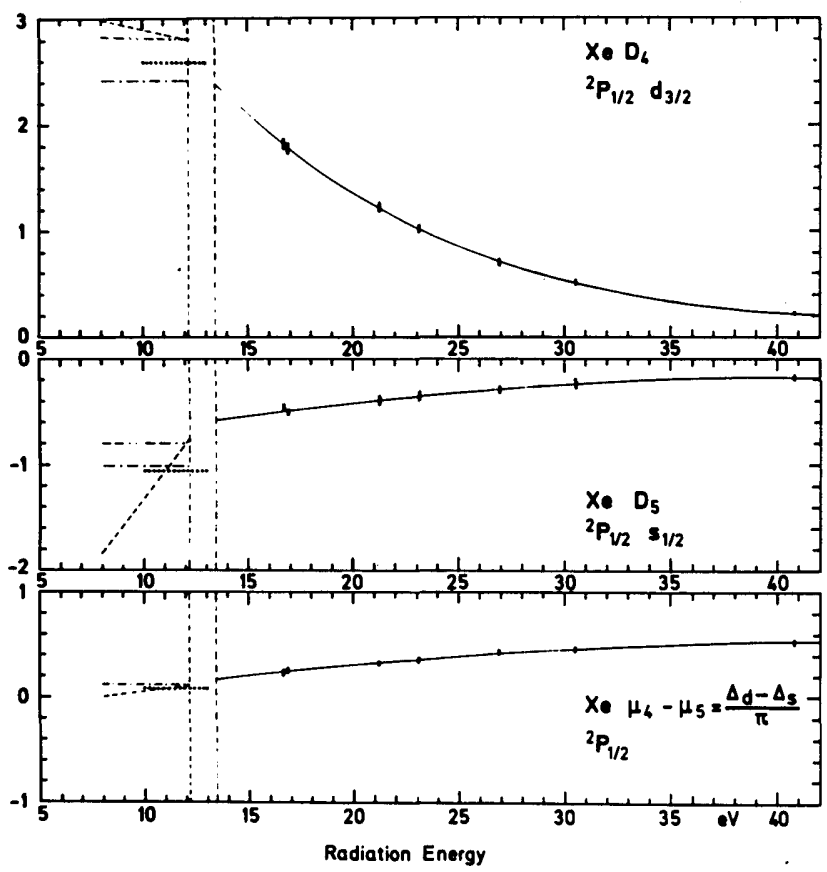

Fig. 7. Photoionization of Xe atoms referring to its outermost $p_{1 / 2}$ subshell. (Top, center) matrix elements determined from experimental results (error bars) ${ }^{11}$ compared with the data by other authors ${ }^{2,12,24}$ in the discrete spectral range on the left-hand side of photoionization thresholds (vertical and dashed lines). (Bottom) phase difference of the continuum wave functions. go into an $s_{1 / 2}$ or $d_{3 / 2}$ continuum state, after the photon has been absorbed by the Xe atom leaving behind a ${ }^{2} P_{1 / 2}$ ion.

The corresponding matrix elements (top and center of Fig. 7) determined experimentally from the thresholds up to $41-\mathrm{eV}$ photon energy are compared with the results obtained by other authors $2,12,24$ in the discrete spectral range, shown in Fig. 7 on the left-hand sides of the two ionization thresholds. The very good agreement in the cross-comparison between the data of photoexcitation in the discrete range and photoionization, also seen in the phase-difference of the wave functions [shown in Fig. 7 (bottom) in units of $\pi$ ] provides new experimental evidence for the validity of the MQDT.

Without going into more detail (for a detailed description see Ref. 11), it should also be noted that in the determination of these matrix elements some new aspects of many electron correlations in Xe have been found in the region of autoionization resonances.

\section{References}

1. N. A. Cherepkov, Sov. Phys. JETP 38, 463 (1974).

2. C. M. Lee, Phys. Rev. A 10, 1598 (1974).

3. U. Fano, Phys. Rev. 178, 131 (1969).

4. U. Heinzmann, G. Schönhense, and A. Wolcke, in Abstracts, Contributed Papers (1978) and Proceedings, International Workshop on Coherence and Correlation in Atomic Collisions, London, H. Kleinpoppen and J. Williams, Eds. (Plenum, New York, 1980), pp. 607-612.

5. U. Heinzmann, G. Schönhense, and J. Kessler, Phys. Rev. Lett. 42, 1603 (1979).

6. U. Heinzmann, G. Schönhense, and J. Kessler, J. Phys. B. 13, L153 (1980).

7. U. Heinzmann and G. Schönhense, III-64, in Extended Abstracts, Sixth International Conference on VUV Radiation Physics, June 1980, and to be published.

8. G. Schönhense, Phys. Rev. Lett. 44, 640 (1980).

9. K. N. Huang, W. R. Johnson, and K. T. Cheng, Phys. Rev. Lett. 43, 1658 (1979).

10. N. A. Cherepkov, J. Phys. B: 12, 1279 (1979).

11. U. Heinzmann, J. Phys. B: 13, in press, (Dec. 1980).

12. J. Geiger, U. Kaiserslautern; private communication (1979).

13. U. Heinzmann,.F. Schäfers, K. Thimm, A. Wolcke, and J. Kessler, J. Phys. B: 12, L679 (1979)

14. J. A. R. Samson, J. Opt. Soc. Am. 54, 6 (1964).

15. U. Heinzmann, B. Osterheld, and F. Schäfers, II-11 in Ref. 7 and to be published.

16. U. Heinzmann and F. Schäfers, J. Phys. B: 13, L415 (1980).

17. U. Heinzmann, B. A. Hess, and F. Schäfers, Chem. Phys. Lett. 69, $284(1980)$.

18. V. Saile, Thesis, U. Munich (1976).

19. R. E. Huffmann, Y. Tanaka, and J. C. Larrabee, J. Chem. Phys. 39, 902 (1963).

20. J. Berkowitz and J. H. D. Eland, J. Chem. Phys. 67, 2740 (1977).

21. R. Frey, B. Gotchev, W. B. Peatman, H. Pollak, and E. W. Schlag, Chem. Phys. Lett. 54, 411 (1978).

22. U. Heinzmann, A. Wolcke, and J. Kessler, J. Phys. B 13, 3149 (1980).

23. U. Heinzmann, J. Phys. E 10, 1001 (1977).

24. J. Geiger, Z. Phys. Abt. A: 276, 219 (1976); 282, 129 (1977). 


\section{Keep informed of new developments in lasers \& electro-optical technologies, $\$ 7$ a year brings you membership in QEAS and 12 issues of JQE.}

The Quantum Electronics Application Society is made up of IEEE members interested in Lasers and Electro-optics. It keeps its members informed by conferences, professional advancement courses, and local chapter seminars, and it publishes a newsletter and the Journal of Quantum Electronics.

You can join the experts and receive all benefits immediately with a mere $\$ 7 /$ year increment in your IEEE membership fee. Please return the form below today.

Name

Address

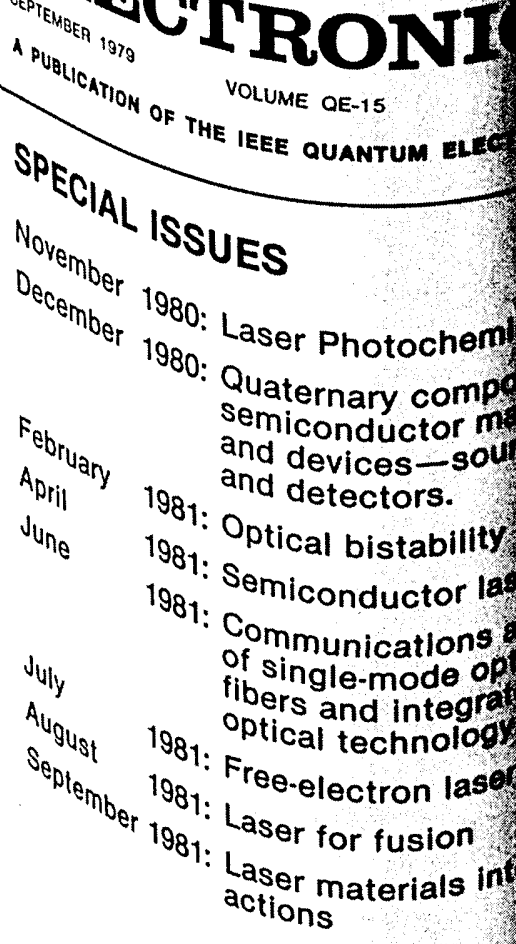

Address

Telephone

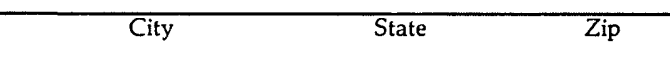

IEEE Members:

Please return this form with $\$ 7.00$ to:

IEEE Service Center

Dept.QEA G/S

445 Hoes Lane

Piscataway, NJ 08854

Membership No.

Non-Members:

Please return this form to:

Dr. Milton Chang

Publicity \& Membership Chairman c/o Newport Research Corporation $18235 \mathrm{Mt}$. Baldy Circle

Fountain Valley, CA 92708

You will receive membership information and application form.
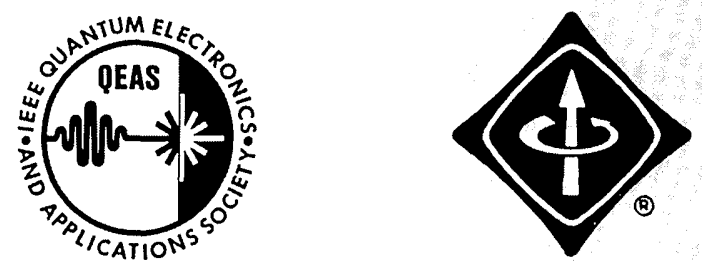